\title{
Les Japygidés cavernicoles de la faune française
}

\author{
Jean PAGÉS*
}

Douze espèces de Japygidés sont actuellement connues de France. Un certain nombre d'entre elles ont été rencontrées dans le domaine hypogé. Cette note a pour but d'en dresser un catalogue critique aussi complet que possible, basé sur les données de la littérature et sur les collections de ces Diploures que m'ont fait parvenir de nombreux naturalistes que je ne saurais trop remercier ici.

Auparavant je crois utile d'exposer dans ses grandes lignes la répartition de ces Insectes dans notre pays. Il est encore malheureusement impossible d'établir avec précision les aires respectives occupées par chacune des douze espèces françaises. Deux raisons permettent, semble-t-il, d'expliquer cet état de chose. Tout d'abord les Japygidés qui vivent normalement dans l'épaisseur des sols, ne forment jamais de colonies denses par suite de l'existence d'un territoire individuel primitif, fluctuant selon les saisons (Pagés, 1967a, 1967b); trés dispersés par conséquent, leur capture ne peut être qu'accidentelle par un non spécialiste de la faune du sol et encore ce dernier n'aura-t-il la chance d'en rencontrer que s'il prospecte un volume de terre assez important; il faudra, de plus, qu'il travaille dans un sol où tout un ensemble de conditions de milieu strictement définies soient réunies (Pagés, 1967a).

De ces difficultés de récolte découle directement la seconde raison expliquant les lacunes de notre documentation sur la chorologie des espèces françaises. En effet, la majorité des naturalistes n'ayant que rarement l'occasion de découvrir des Japyx, ils s'empressent d'étudier "égoïstement" leur capture sans se douter de l'intérêt plus général de leur prise.

Ces réserves étant faites on peut dire qu'en France l'espèce dominante est Dipljapyx humberti (Grassi) qui a été trouvée sur un territoire extérieurement limité par une ligne passant approximativement par Brest-Narbonne-littoral méditerranéen-Nice-Genève-Francfort-Liège; il est remarquable de constater qu'aucune station n'est connue du bassin de la Garonne; je ne l'ai pas non plus rencontrée en Meurthe-et-Moselle malgré de très nombreuses prospections.

Deux autres espèces doivent aussi être assez largement répandues: Metajapyx leruthi (Silv.) à l'Est d'une ligne Liège-Banyuls-Bâle et Protjapyx major (Grassi) qui n'est actuellement connue que d'une demi-douzaine de stations où elle a dû être importée par l'Homme (jardins, serre, etc....) dans la région parisienne, à Strasbourg, en Côte-d'Or et dans L'Hérault.

Les neuf autres espèces sont localisées dans les régions méridionales et peuvent être réparties en deux groupes.

A l'Est du Rhône se rencontre une faune à affinités italiennes: Japyx solifugus Hal., Unjapyx simplicior (Silv.), Metajapyx bonadonai Pagés, Dipljapyx fagniezi

* Laboratoire de Biologie Animale et Générale, Faculté des Sciences de la Vie et de l'Environnement, 6, Bd. Gabriel, 21-DIJON, France. 
Pagés, auxquelles il faut ajouter Unj. clayi Pagés et Diplj. beroni Pagés de Corse.

A l'Ouest du Rhône, approximativement à partir de $500 \mathrm{~m}$ d'altitude le long des Cévennes et des Pyrénées, trois espèces à affinités espagnoles: Metaj. codinai (Silv.), Metaj. doderoi (Silv.) et Metaj. gallicus (Silv.).

En ce qui concerne le domaine hypogé nos connaissances sont aussi peu fournies car, si pratiquement tous les spécimens récoltés ont fini par parvenir à un spécialiste, la probabilité de rencontrer des Japyx se promenant à la surface du sol, bien qu'un peu plus grande que dans le domaine épigé, est cependant très faible (Pagés, 1967a). C'est une des raisons qui m'ont fait inclure dans la liste suivante des captures faites à l'entrée de grottes par lavage de terre.

\section{LISTE DES FORMES}

\section{Japyx indéterminé.}

Catacombes de Paris, 1 ex., Viré (1896). J'ai dit dans une note antérieure (1951) que cet exemplaire n'est certainement pas un Japyx solifugus Hal. comme l'écrit Houlbert (1924), mais étant perdu, rien ne permet d'avancer un nom quelconque; deux espèces, Diplj. humberti et Protj. major peuvent se rencontrer dans la région parisienne.

Grotte de la Foux (Var), Chappuis et Jeannel (1951). Dans la très courte description de cette cavité il est écrit: “c”est un long ruisseau souterrain à sec, avec grandes accumulations d'argile sur lesquelles le Japyx était abondant". Il s'agit très certainement d'une lapsus car le matériel en provenance de cette grotte (Matériel No. 1356, août 1929) que j'ai pu examiner ne renferme aucun Japyx mais des Nicoletia jeanneli Silv.; Silvestri qui a décrit cette espèce (1938) sur quatre exemplaires de cette localité cite une lettre de Jeannel qui dit "que le Thysanoure se trouve çà et là errant sur les nappes d'argile".

Il n'est pas impossible que, comme dans les Catacombes de Paris, l'on trouve un jour des Japyx dans cette grotte, mais actuellement cette station ne peut être prise en considération.

\section{Japyx solifugus Haliday}

Cette espèce est celle que l'on cite traditionellement quand on parle des Japygidés alors que, mise à part l'Italie, elle ne soit, ni très répandue, ni très commune. En France sa station la plus septentrionale et la plus occidentale que je connaisse est située au Sud de Montélimar; il s'ensuit que tout $J$. solifugus signalé hors de cette zone, limitée par le Sud du massif alpin, Le Rhône et la frontière italienne, n'en est pas un.

Catacombes de Paris, Houlbert 1924). J'ai dit dans le paragraphe précédent ce qu'il fallait penser de cette détermination.

Traou de Guille près de Digne (Basses-Alpes), 1 ex., Peyerimhoff (1906). Il est possible que cet exemplaire soit bien un solifugus, mais n'ayant, ni revu ce spécimen, ni examiné de nouvelles captures en provenance de cette localité, je ne puis ni le certifier, ni proposer un autre nom. Comme on le verra ci-dessous tous les 
Japyx cavernicoles jusqu'à présent connus de cette région sont des Diplj. fagniezi Pagés.

\section{Metajapyx codinai (Silv.).}

Cette espèce décrite d'Espagne est commune dans le domaine endogé pyrénéen et a déjà été signalée deux fois dans des grottes:

Grotte de Gourgues, Arbas (Haute-Garonne), 1 ex., Silvestri (1934).

Grotte d'En Brixot, près de la Preste (Pyrénées-Orientales), 1 ex., Pagés (1952).

A ces deux stations je puis ajouter les suivantes grâce à l'abondant matériel que m'a fait parvenir H. Coiffait; ces stations sont cependant à la limite du domaine hypogé puisqu'il s'agit de lavages de terre faits à l'entrée des grottes.

Entrée de la grotte du Castel de l'Estelle, Cabanac-Cazaux (Haute-Garonne), 8 ex.

Grotte de Gourgues, talus d'entrée, Arbas (Haute-Garonne), 6 ex.

Entrée de la grotte du Goueil di Her, dans les mouses, Arbas (Haute-Garonne), 1 ex.

Grotte de Lestelas, talus d'entrée, Cazavet (Ariège), 4 ex.

Entrée de la grotte du Cap del Bouich, Moulis (Ariège), 1 ex.

Entrée de la grotte du Tuc d'Audoubert, Montesquieu-Avantès (Ariège), 2 ex.

Lieudit Rougé, au fond d'une doline, Riverenert (Ariège), 2 ex.

Entrée de la grotte de Hount Santo, Ustou (Ariège). 1 ex.

Entrée du Goueil de Jouéou, dans les mousses, Val d'Aran, 1 ex. Soit un total général de 26 individus pour ces neuf stations.

4. Metajapyx doderoi (Silv.).

Cette espèce n'est connue que par une unique femelle capturée dans:

Grotte de Castel-Mouly, Bagnères de Bigorre (Htes-Pyrénées), Silvestri (1934).

\section{Metajapyx gallicus (Silv.)}

Décrit par Silvestri (1934) d'après trois exemplaires pris dans les cavités suivantes:

Grotte de Sainte-Hélène, Foix (Ariège), 1 ex.

Grotte de Rieufourcau, Belesta (Ariège), 1 ex.

Grotte de Fontanet, Ornolac (Ariège), 1 ex.

J'ai retrouvé cette espèce dans les deux localités suivantes où elle a été prise par

H. Coiffait dans des biotopes identiques à ceux de Metaj. codinai:

Grande grotte de Labastide, talus d'entrée, Labastide (Htes-Pyrénées), 1 ex.

Entrée de la grotte de l'Haiouat de Pelou, Haut Nistos (Htes-Pyrénées) 2 ex.

Il est remarquable de constater que ces nouvelles stations sont séparées des plus anciennes par de nombreuses localités épigées et hypogées où seul vit Metaj. codinai.

\section{Dipljapyx beroni Pagés.}

Cette espèce, endémique en Corse, est très proche de Diplj. humberti et de Diplj. italicus (Silv.) dont elle se distingue sans peine par ses antennes de 31 articles; elle vient d'être récoltée dans deux grottes corses par P. Beron: 
Grotte de Manuel-Ange, presqu'ile d'Orchino, au Nord de Cargèse, 2 ex.

Grotte de Grottone, vers Lama, 1 ex. Je la connaissais auparavant d'une station épigée.

\section{Dipljapix fagniezi Pagés}

Décrite d'après cinq spécimens provenant de:

Grotte Mars, Vence (Alpes-Maritimes), Pagés (1952).

Je viens de la retrouver dans du matériel que m'a fait parvenir P. Bonadona:

Grotte de Peygros, dans la doline, Fayence (Var), 1 ex.

Elle est présente dans d'autres stations épigées prospectées par ce naturaliste.

\section{Protjapyx major (Grassi)}

Déjà signalé des deux cavités suivantes:

Catacombes du Muséum national d'Histoire naturelle de Paris, 1 ex., Pagés (1951).

Grotte du Figuier, Minerve (Hérault), 1 ex., Pagés (1967a).

Cette espèce est répandue dans tous les pays bordant la Méditerranée occidentale.

La liste précédente, malgré le nombre relativement restreint de captures, permet d'énoncer un certain nombre de conclusions générales.

1. Six espèces sont donc connues avec certitude du domaine hypogé: Metaj. codinai, M. doderoi, M. gallicus, Diplj. beroni, D. fagniezi, Protj. major. Il faut peut-être y ajouter $J$. solifugus, mais en se basant uniquement sur le fait que le spécimen de la région de Digne a été capturé dans la zone où peut se rencontrer cette espèce.

2. Metajapyx doderoi est le seul à n'être connu que du domaine hypogé, mais rappelons le par un seul spécimen et il n'a jamais été retrouvé depuis sa description en 1934.

3. En Europe des Japyx ont également été signalés dans des grottes en Yougoslavie, Italie et Espagne. En ne tenant compte que des espèces dont la détermination est certaine on constante que dans ces différents pays les genres à espèces cavernicoles sont:

Metajapyx en Yougoslavie, Italie, France, Espagne

Dipljapyx en Italie et en France

Protjapyx en France et en Espagne

Monojapyx et Homojapyx en Espagne

Il paraît bien difficile à l'heure actuelle, compte tenu des données très fragmentaires sur la composition exacte de la faune japygidienne de ces quatre pays et surtout sur la répartition précise de chaque espèce, d'expliquer cet appauvrissement de l'Ouest à L'Est. Peut-être faut-il chercher une solution dans le fait que certains Japygidés seraient plus aptes que d'autres à supporter des températures relativement bases; $D$. humberti par exemple ne pourrait que rarement être cavernicole en France puisqu'en dessous de $10^{\circ} \mathrm{C}$ son cycle vital ne peut se fermer (Pagés, 
1967a). Ce dernier fait peut permettre aussi d'envisager pour comprendre cette distribution l'influence desglaciations sur le climat de l'Europe. Mais cette dernière hypothèse, en l'absence de toute trace fossile, nécessitera de minutieuses recherches tant du point de vue biologique que de la morphologie avant de pouvoir être efficacement vérifiée.

4. Aucun des Japyx cavernicoles français ne montre la moindre modification morphologique que l'on puisse attribuer à la vie cavernicole et tous, sauf $M$. doderoi, ont été capturés aussi bien dans le domaine endogé.

La discrimination sélective qui semble s'instaurer entre les nombreuses espèces de Japygidés m'avait incité (Pagés, 1964) à considérer comme des troglophiles celles qui peuplent le domaine souterrain; cependant ce terme est trop précis dans ses implications biologiques (Racovitza, 1907) et ce n'est qu'avec difficultés que l'on peut l'adapter au cas des Japygidés cavernicoles et en particulier à ceux de France.

Ces derniers ne peuvent pas, à mon sens et compte tenu de nos connaissances actuelles, être considérés seulement comme des endogés édaphobies (cf. Coiffait, 1958) rencontrés par hasard dans les grottes, donc assimilés aux trogloxènes. Certains se révèleront sûrement l'être comme par exemple Protj. major, mais la majorité d'entre eux montreront toujours une tendance à vivre dans les cavernes. On pourraît alors leur appliquer le qualicatif de subtroglophile tel qư il est défini par Pavan (1944) et Ruffo (1955). Cependant des auteurs récents (Motas et all., 1967 par exemple), étudiant la faune de l'association pariétale considèrent que l'état subtroglophile est lié presque toujours a une diapause que subissent à un moment quelconque de leur cycle biologique les espèces rencontrées dans ce biotope. Ce n'est certainement pas le cas des Japygidés chez lesquels une vraie diapause n'a jamais été rencontrée (Pagés, 1967a); il ne faut donner dans ce cas au mot subtroglophile aucune implication biologique autre que celle de constater que certaines espèces se rencontrent dans les grottes et d'autres pas. *

\section{RÉ S U M É}

Les Japygidés sont représentés en France par 12 espèces dont 6, déterminées avec certitude, ont été récoltées dans les grottes ou à l'entrée de celles-ci. Aucune ne montre de modifications morphologiques imputables à la vie cavernicole. Toutes, sauf Metaj. doderoi Silv. connue par une seule $q$ de la grotte de Castel Mouly, se retrouvent dans le domaine épigé.

Pour tenir compte de la sélection qui semble cependant s'effectuer entre les espèces, le terme de subtroglophile est proposé pour qualifier celles qui peuvent se rencontrer dans le domaine hypogé.

\section{S U M M A R Y}

Twelve species of Japygids are cited from France. Six are known with certitude

* Note présentée au 8e Congrès national de Spéléologie (Draguignan, 1968). Un résumé succinct en a été publié dans les comptes rendus de ce Congrès (1969). 
from caves. None of them shows any morphological modification which can be ascribed to cavernicolous life. All of them are found outside caves except Metaj. doderoi Silv. which is known from a unique $q$ from Castel Mouly cave.

To take into account the selection which seems to occur among the species, the term "subtroglophile" is proposed to include those which can be found in the caves.

\section{B I B L I O G R A P H I E}

CHAPPUIS, P.-A. et JEANNEL, R., 1951 - Enumération des grottes visitées, 1927-1949 (Huitième série). Biospeologica LXXII. Arch. Zool. exp. gén., 88, 81-230.

COIFFAIT, H., 1958 - Les Coléoptères du sol. Vie et Milieu, suppl. No. 7, 204 pp.

HOULBERT, C., 1924 - Thysanoures, Dermaptères et Orthoptères. Encyclopédie scientifique, Doin éd., Paris.

MOTAS, C., DECOU, V. et BUYGHELE, A., 1967 - Sur l'association pariétale des grottes d'Olténie (Roumanie). Ann. Spéléo., 22, 474-522.

PAGES, J., 1951 - Un Diploure Japygidé des Catacombes de Paris. Bull. Mus. nat. Hist. nat. Paris, (2), 23, 217-219.

PAGES, J., 1952 - Un Diploure Japygidé inédit de la grotte Mars (Alpes-Maritimes). Notes biosp., 7, 69-74.

PAGES, J., 1964 - Remarques sur les Japygidae (Insecta, Diplura) signalés dans le domaine souterrain. Int. J. Speleol., 1, 191-201.

PAGES, J., 1967a - Données sur la biologie de Dipljapyx humberti (Grassi). Rev. Ecol. Biol. Sol., 4, 187-281.

PAGES, J., $1967 b$ - La notion de territoire chez les Diploures Japygidés. Ann. Soc. ent. Fr., (N.S.), 3, 715-719.

PAGES, J., 1969 - Les Japygidés cavernicoles de la faune française. Spelunca (4), Mém. No. 6, Actes du 8e Congr. nat. Speleo., p. 88.

PAVAN, M., 1944 - Considerazioni sui concetti di troglobio, troglofilo e troglosseno. Le Grotte d'Italia, (2), 5, 35-41.

PEYERIMHOFF, P., 1906 - Recherches sur la faune cavernicole des Basses-Alpes. Ann. Soc. ent. Fr., 75, 203-233.

RACOVITZA, E.G., 1907 - Essai sur les problèmes biospéologiques. Biospeologica I. Arch. Zool. exp. gén., (4), 6, 371-488.

RUFFO, S., 1955 - Le attuali conoscenze sulla fauna cavernicola della regione pugliese. Mem. Biogeogr. Adriatica, 3, 143 pp.

SILVESTRI, F., 1934 - Dicellura, Japygidae, Biospeologica LXI. Arch. Zool. exp. gén., 76, 385-398.

SILVESTRI, F., 1938 - Descrizione di una nuova specie di Nicoletia vivente in una grotta della Francia. Rev. fr. Ent., 5, 188-193.

VIRE, A., 1896 - La faune des Catacombes de Paris. Bull. Mus. Hist. nat. Paris, 2, 226-234. 